\title{
Digitales Röntgen für die Arztpraxis
}

Markus Dalcher, Roland Koch, Andreas Stalder

Kliniken und Röntgeninstitute der Schweiz haben den Technologiewandel bei der Projektionsradiographie von den konventionellen Folien-Film-Systemen zu den digitalen Aufnahmeverfahren weitgehend vollzogen. Digitale Aufnahmeverfahren bieten den Vorteil, dass Kontrast und Helligkeit des «Rohbildes» organspezifisch nachbearbeitet werden können. Digitale Filtertechnik ermöglicht die Verbesserung der Detailerkennbarkeit, und durch die Wahl der Tiefe und der Lage des digitalen Bildfensters können Über- wie auch Unterexpositionen teilweise kompensiert werden. Zuweiser werden mit Drucken auf Laser- oder Thermofilmen bedient. Auf Wunsch werden Patienten-CDs mit Viewingsoftware oder digitale Bildübermittlungsverfahren zur Betrachtung auf dem PraxisPC angeboten.

\section{Verfahren und Anwendung: \\ CR (Computed Radiography) oder Speicherfolientechnologie}

Mehr als 95\% der digitalen Röntgenaufnahmen werden mittels digitaler Speicherfolientechnologie hergestellt. Anstelle einer Röntgenkassette mit Verstärkerfolie und Röntgenfilm erfolgt die Aufnahme auf einer Kassette mit Speicherfolie. Die Speicherfolie besitzt die Eigenschaft, ein Bild solange latent zu speichern, bis die Folie unter Einfluss von Laserenergie Punkt für Punkt (Pixel für Pixel) eine Lichtmenge aussendet, die der absorbierten Röntgenstrahlung entspricht. Das emittierte Licht wird jedem Pixel der Bildmatrix zugeordnet und es entsteht ein digitales Rohbild, das zur automatischen, organspezifischen Aufbereitung an den Bildrechner übermittelt wird. CR-Systeme sind meistens in ein PACS (Picture Archiving and Communication System) eingebunden, welches seinerseits zur Übernahme von administrativen Daten mit dem RIS (Radiologie Informations-System) kommuniziert.

\section{Ablauf einer Speicherfolienaufnahme}

\section{Gerätebedarf}

Mit jedem praxisüblichen Röntgengerät kann anstelle einer normalen Röntgenkassette mit Folien und Film eine Kassette mit Speicherfolie verwendet werden. Vor der Aufnahme erfolgt die Identifikation der Kassette mit den Personalien des Patienten und dem Hinweis auf das zu röntgende Organ. Bei Extremitätenaufnahmen erfolgt die Exposition direkt auf die Kassette, bei Aufnahmen an Schädel und Körperstamm wird die Kassette zur Exposition in die Buckyblende eingelegt. Als Expositionsdaten kommen diejenigen für die Empfindlichkeitsklasse 200-400 zur Anwendung. Nach erfolgter Aufnahme wird die Kassette in den Speicherfolienscanner eingelegt, der Auslesevorgang beginnt. Auf einem Viewingmonitor kann der Bildaufbau in Rohqualität mitverfolgt werden. Sind alle Rohdaten vorhanden, wird unter Berücksichtigung der organspezifischen Gradiationskurve und vorgewählten Filter ein optimales Bild errechnet, welches auf dem Viewingmonitor erscheint und automatisch oder manuell patienten- und untersuchungsspezifisch gespeichert wird. Für die Befundung wird das Bild entweder zu einer Workstation mit Befundmonitor gesendet oder es wird mit einem Laser- oder Thermoprinter auf Film gedruckt. An der Workstation können die digitalen Bildparameter und Filter dem Bedarf angepasst werden. Der Ausdruck auf Film kann verkleinert oder 1:1 erfolgen.

Neben der bestehenden oder einer neuen Röntgenanlage werden für die Speicherfolientechnologie ein Speicherfolienscanner mit Patientenidentifikation und Viewingmonitor sowie einige Kassetten benötigt; dazu entweder ein Monitor in Befundungsqualität oder ein Dicomfähiger Laser- oder Thermodrucker für die Bildwiedergabe auf Film. Digitale Aufnahmeverfahren verlangen ebenfalls nach den Massnahmen der Qualitätssicherung wie das konventionelle Röntgen. 


\section{Kosten}

Wer sich mit der Anschaffung eines Speicherfoliensystems beschäftigt, der darf heute in seiner Kalkulation von einem Investitionsvolumen von mindestens Fr. 150000.- ausgehen (ohne Röntgenanlage). Die jährlich wiederkehrenden Kosten für Hotline, Service, Reparaturen und Qualitätssicherung dürften je nach Umfang der Leistungen 5-10\% der Investition ausmachen. Wenn alle Bilder ausgedruckt werden, sind auch die Filmkosten mitzurechnen. Aus diesem Grunde eignen sich Speicherfoliensysteme der heutigen Generation in erster Linie für Arztpraxen mit grossem Röntgenaufkommen oder für Gemeinschaftspraxen.

\section{Andere digitale Verfahren}

Flat-Panels, DR (Digital Radiography) oder Flächendetektoren

Sie enthalten einen $35 \times 43 \mathrm{~cm}$ grossen $« \mathrm{CCD}$ Chip». Sie kommen in Kliniken zur Anwendung, in denen mit ein- und demselben Bildempfänger grosse Zahlen von Röntgenaufnahmen erstellt werden. Jeder Röntgentisch oder jedes Wandstativ muss mit einem Flat-Panel ausgerüstet werden oder es kommt ein C-Bogen-ähnliches Gerät zum Einsatz. Diese sind bedeutend teurer als CR-Systeme, aber rationeller im Arbeitsablauf.

\section{Linear-Scanning-Technologie}

Eine Röntgenröhre mit Schlitzfenster als Strahlenquelle und auf der Aufnahmeseite auf einer Linie angebrachte Detektoren in der Anzahl Pixel der X-Achse bewegen sich während der Aufnahme in Richtung der Y-Achse der Bildmatrix. Örtlich kurze, aber für den ganzen Scanvorgang längere Expositionszeiten sind die Folge. Diese Technologie steht im Moment vor der Markteinführung.

\section{Systeme in Entwicklung}

Vollautomatische, im Buckystativ integrierte, kassettenlose Speicherfoliensysteme sind in Entwicklung.

Alle technischen und die Preisangaben sind unverbindliche Informationen. 